\title{
Erratum to: Phosphorus fractions and bioavailability in relation to particle size characteristics in sediments from Lake Hongfeng, Southwest China
}

\author{
Yuanrong Zhu $\cdot$ Runyu Zhang $\cdot$ Fengchang Wu \\ Xiaoxia Qu $\cdot$ Fazhi Xie $\cdot$ Zhiyou Fu
}

Published online: 19 January 2013

(c) Springer-Verlag Berlin Heidelberg 2013

\section{Erratum to: Environ Earth Sci}

DOI 10.1007/s12665-012-1806-9

The authors would like to correct errors in the original publication as detailed below:

The author affiliations of R. Zhang and F. Xie should be corrected as:

\section{R. Zhang}

State Key Laboratory of Environmental Geochemistry, Institute of Geochemistry, Chinese Academy of Sciences, Guiyang 550002, China

The online version of the original article can be found under doi:10.1007/s12665-012-1806-9.

Y. Zhu $\cdot$ X. Qu

College of Water Sciences, Beijing Normal University,

Beijing 100012, China

Y. Zhu · F. Wu $(\bowtie) \cdot$ X. Qu $\cdot$ F. Xie $\cdot$ Z. Fu

State Key Laboratory of Environmental Criteria and Risk

Assessment, Chinese Research Academy of Environmental

Sciences, Beijing 100012, China

e-mail: wufengchang@vip.skleg.cn

\section{R. Zhang}

State Key Laboratory of Environmental Geochemistry,

Institute of Geochemistry, Chinese Academy of Sciences,

Guiyang 550002, China

F. Xie

CAS Key Laboratory of Crust-Mantle Materials

and Environment, School of Earth and Space Sciences,

University of Science and Technology of China,

Hefei 230026, China
F. Xie

CAS Key Laboratory of Crust-Mantle Materials and Environment, School of Earth and Space Sciences, Hefei 230026, China 